\title{
Influence of Solutes on Hydration and Lubricity of Dextran Brushes
}

\author{
Tolga Goren ${ }^{\S a}$, Rowena Crockett ${ }^{\mathrm{b}}$, and Nicholas D. Spencer ${ }^{\star a}$ \\ §SCS-Metrohm Foundation Award for best oral presentation
}

\begin{abstract}
The characteristic lubricity and non-fouling behavior of polymer brushes is critically dependent on the solvation of the polymer chains, as well as the chain-chain interactions. Dextran brushes have shown promise as non-toxic aqueous lubricant films, and are similar in composition to natural lubricating systems, while their comparative simplicity allows for controlled preparation and fine characterization. This project entails measuring the solvation and lubricity of dextran brushes in the presence of additives which modify the inter-chain hydrogen bonding. The thickness and refractive index of the film were measured during adsorption of the brush layer onto a silica substrate and the subsequent immersion in solutions of potassium sulfate and $\alpha, \alpha$-trehalose. We also studied the lubricity of the system as a function of normal loading using colloidal-probe AFM. Both solutes are shown to have a minimal effect on the hydration of the brush while significantly reducing the brush lubricity, indicating that inter-chain hydrogen bonding supports the load-bearing capacity of polysaccharide brushes.
\end{abstract}

Keywords: Aqueous lubrication · Dextran · Friction · Hydration · Polymer brush

\section{Introduction}

Polymer brushes have shown great promise as lubricious surface coatings for aqueous tribosystems. ${ }^{[1-3]}$ Composed of densely packed end-attached polymer chains, their high degree of solvation and load-bearing capacity act to reduce friction and wear, even in boundary-lubrication conditions, where the hydrodynamic fluid film alone would be insufficient to maintain separation between opposing asperities. When the brush is comprised of nontoxic polymers, such as PEG or dextran, the resulting system has great potential in the fields of food and drug processing, biomedical engineering, and microfluidics. ${ }^{[4]}$

The study of polymer brush-mediated aqueous tribosystems is also interesting because they share some common properties with naturally occurring lubricious

${ }^{*}$ Correspondence: Prof. Dr. N. D. Spencera

Tel.: +4144632 5850

Fax: +41446331027

E-mail: nspencer@ethz.ch

aLaboratory for Surface Science and Technology

ETH Zurich

Wolfgang-Pauli-Strasse 10

$\mathrm{CH}-8093$ Zürich

'EMPA, Swiss Federal Institute for Materials Science and Technology

Überlandstrasse 129

$\mathrm{CH}-8600$ Dübendorf systems, such as the glycocalyx, ${ }^{[5]}$ articular cartilage joints, ${ }^{[6]}$ and slippery surfaces such as the tongue, ${ }^{[7]}$ which are comprised of densely arranged oligosaccharide-bearing biomolecule-coated surfaces separated by aqueous lubricants such as blood plasma, synovial fluid, or mucus. The complexity and sensitivity of these systems render the study of their mechanisms of lubrication highly challenging. Polymer- and especially polysaccharide-based brushes represent simpler, homogeneous systems that capture some of the critical aspects of natural lubrication.

Dextran is a naturally occurring glucan with high water solubility. The orientation and degrees of freedom conferred by its $(1 \rightarrow 6)$ linkage hinder the formation of stable lamellar structures by the relatively flexible chains, making it suitable for the formation of brushes. Poly(L-lysine)graft-dextran (PLL- $g$-dex $)^{[8,9]}$ is a bottlebrush copolymer formed by grafting some of the primary amine groups of a poly $(\mathrm{L}-$ lysine) backbone to the terminal aldehydes of dextran chains via reductive amination. The unreacted amines maintain a positive charge in aqueous solution at neutral $\mathrm{pH}$, and the backbone will spontaneously adsorb onto negatively charged surfaces at multiple locations, while the uncharged, hydrophilic dextran chains extend into solution. On silicon oxide surfaces in water, these molecules adsorb spontaneously with sufficient density that the dextran inter-chain spacing is significantly lower than its radius of gyration, forcing the chains into a brush conformation.
Dextran brushes have been shown to have non-fouling properties and to be highly lubricious at low loads. ${ }^{[9,10]}$ Above a critical load, however, the friction increases sharply. ${ }^{[10]}$ This critical load is attributed to the point at which the adjacent chains of the brush are forced apart, disrupting the hydrogen bonds, which may then stick to the counterface. Tribosystems composed of opposing dextran brushes show lower friction at low loads, a higher critical load, and higher friction above the critical load than dextran brushes against bare silica. Similar behavior can be seen in models of brushbrush interaction where the chains are long and flexible enough to entangle..$^{[1-14]}$ This is not possible for the shorter, stiffer dextran chains studied, but the inter-chain hydrogen bonds are hypothesized to cause this similar behavior.

In order to better understand the role of inter-chain hydrogen bonding in polysaccharide brushes, we measured the lubricity and swelling of the brush in the presence of solutes that influence hydrogen bonding. Potassium sulfate is an ionic kosmotrope that can screen electrostatic interactions and reduce hydrogen bonding by enhancing dextran's affinity to water. $[15,16]$ Mono- and disaccharides, such as glucose and $\alpha, \alpha$-trehalose, can also reduce interchain cohesion by acting as 'zippers', as they bridge dextran chains and their mobility allows increased freedom to restructure the hydrogen bonds. ${ }^{[17,18]}$ These and similar low-molecular-weight saccharides and polyols are often used as a plasticizers in polysaccharide melts. Trehalose is ex- 


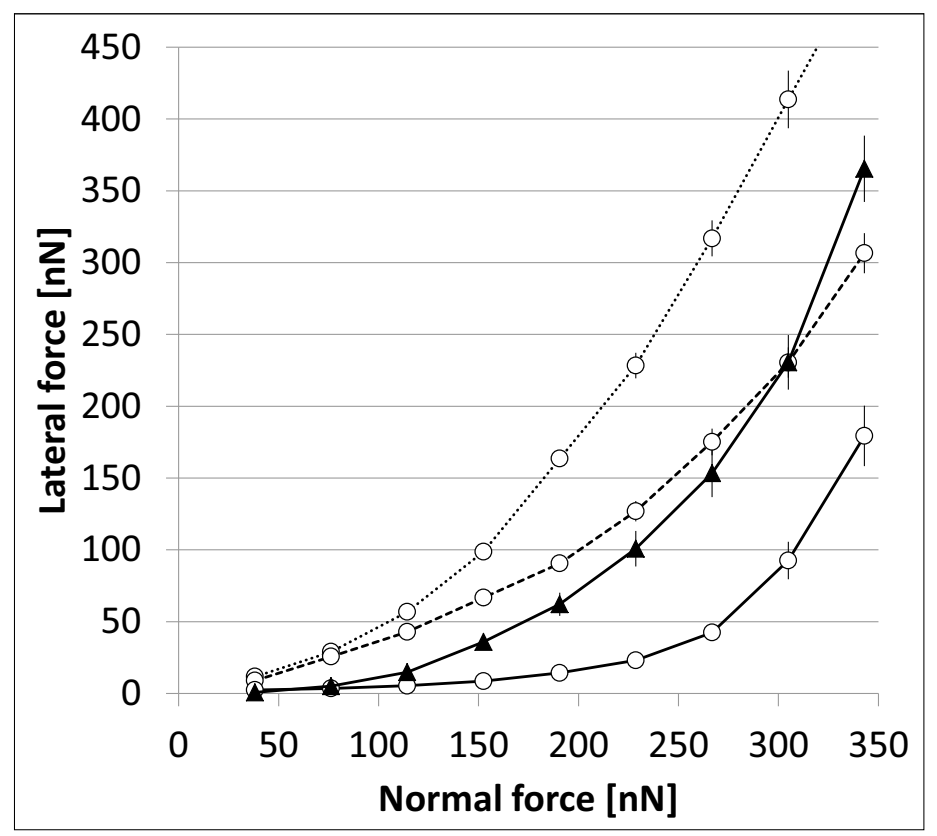

Fig. 1. Friction force measurements of a colloidal probe sliding on a silicon wafer coated with PLL-gdextran at a grafting density of $L / 2 R_{\mathrm{g}}=$ 1.04 (dotted line), $\mathrm{L} / 2 \mathrm{R}_{\mathrm{g}}=0.68$ (dashed line), and $\mathrm{L} / 2 \mathrm{R}_{\mathrm{g}}=0.5$ (solid lines), in ultrapure water $(\mathrm{O})$ and in $5.5 \mathrm{mM}$ trehalose $(\mathbf{\Delta})$.

tremely hydrophilic, with a hydration shell four times as large of that of glucose, and a strong kosmotrope, making it a powerful bioprotectant against plant dehydration. ${ }^{[19]}$ The friction, and especially the transition load, of the dextran brush in the presence of these solutes should be a strong indicator of their effects on the inter-chain hydrogen bonding.

\section{Results and Discussion}

\section{Brush Solvation}

The sensors of two different optical instruments, the Optical Waveguide Lightmode Spectroscope (OWLS) and the Transmission Interference Adsorption Sensor (TInAS), ${ }^{[20,21]}$ were exposed to a solution of PLL-g-dex $(5 \mathrm{kDa})$ in ultrapure water, and the thickness and refractive index of the film were measured in real time during adsorption as the brush was formed. The adsorbed mass of the polymer on the surface, not including any associated water (the 'dry mass'), were calculated from the thickness and refractive index. The two instruments generally show excellent agreement, in this case reporting the maximum adsorbed mass of $306 \pm 15 \mathrm{ng} / \mathrm{cm}^{2}$, corresponding to an average inter-chain distance $\mathrm{L}$ of $1.9 \mathrm{~nm}$ or a grafting density $\mathrm{L} / 2 \mathrm{R}_{\mathrm{g}}$ of 0.5 , taking the radius of gyration $\mathrm{R}_{\mathrm{g}}$ to also be $1.9 \mathrm{~nm} .{ }^{[22]}$ The measurements indicate a mean solvated brush thickness of $7.1 \mathrm{~nm}$ with a refractive index of 1.42 , although these values have a higher mutual uncertainty than their combined optical mass. [21]

After adsorption had slowed down to within the drift of the instruments, the samples were first rinsed with ultrapure water and then exposed to a solution of either 10 $\mathrm{mM}$ potassium sulfate or $5.5 \mathrm{mM}$ trehalose in ultrapure water. The brush showed no measurable change in thickness or refractive index in the presence of either solute, indicating that the solvent quality did not significantly decrease, which would cause the brush to collapse, nor did the solute cause any measurable desorption of the copolymer.

\section{Aqueous Lubricity}

Silicon wafers were incubated in the same PLL-g-dex solution for varying times to achieve different chain densities. They were subsequently rinsed and dried, and the thickness of the film measured with Variable Angle Spectroscopic Ellipsometry (VASE) to determine the adsorbed mass and the homogeneity of the films. Finally, the friction force was measured as a function of load by means of lateral force microscopy, between the brushes and a borosilicate colloidal sphere glued to the cantilever tip. Measurements were performed in ultrapure water and subsequently in the appropriate solution.

\section{Influence of Grafting Density}

Fig. 1 shows the friction response as a function of applied load of PLL- $g$-dex films of different densities. In good agreement with an earlier study, ${ }^{[10]}$ the local coefficient of friction, $\mu=\mathrm{dF}_{\text {lat }} / \mathrm{dF}_{\text {norm }}$, is lowest at low loads, but increases sharply above a critical load. Higher dextran grafting density results in lower friction at low loads and a higher critical load. This critical load is the value at which the applied load partially overcomes the inter-chain bonds and steric forces that force the chains into a brush configuration. Above this load, phenomena such as plowing and dragging overcome the tendency of the brush to maintain a lubricious aqueous film at the surface, resulting in sharply increased friction.

\section{Influence of Solutes}

The friction response of the densest dextran brush in the presence of $5.5 \mathrm{mM}$ trehalose solution was similar to that in ultrapure water at the lowest loads, but continuously increased with increasing load, exhibiting a less well-defined critical load (Fig. 1). At the highest normal force measured in the presence of trehalose, the friction force was around double that in ultrapure water. This is consistent with our prediction that the trehalose acts as a plasticizer for the dextran chains, easing their separation and thus causing a reduction in load-bearing capacity.

The influence of potassium sulfate on the friction of the densest dextran brush is similar to that of trehalose, as shown in Figs. 2 and 3. The friction force and local friction coefficient are similar to the

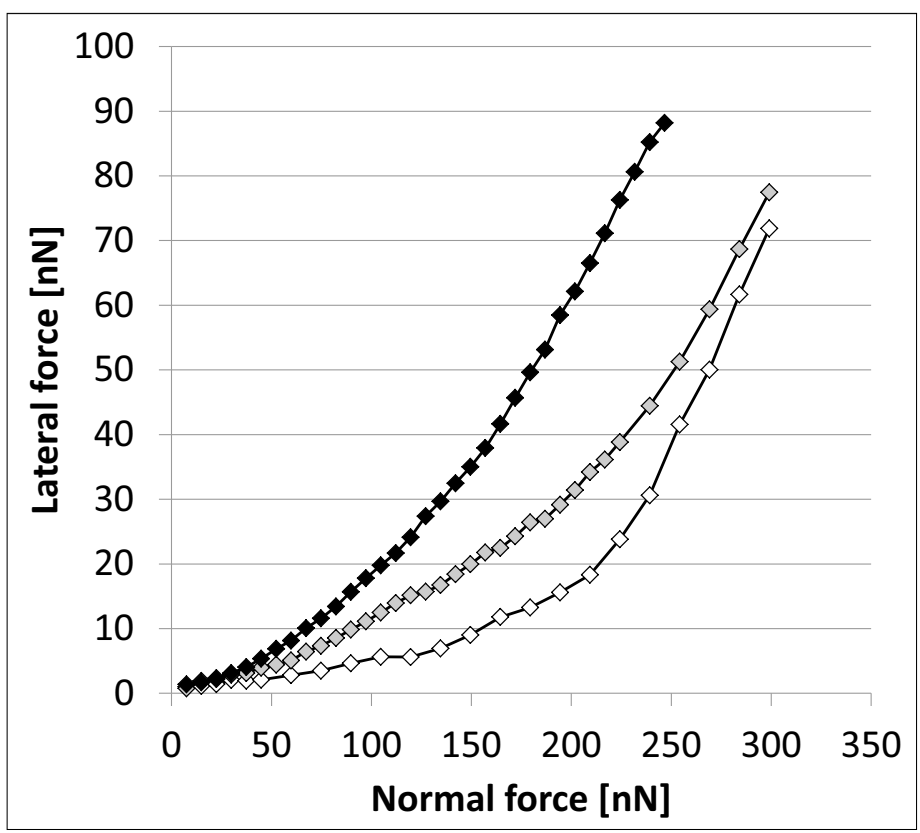

Fig. 2. Friction force measurements of a colloidal probe sliding on a silicon wafer coated with PLL-gdextran at a grafting density of $L / 2 R_{g}=0.5$ in ultrapure water $(\diamond)$, $2 \mathrm{mM} \mathrm{K}_{2} \mathrm{SO}_{4}(\diamond)$, and $10 \mathrm{mM} \mathrm{K}_{2} \mathrm{SO}_{4}(\diamond)$. 


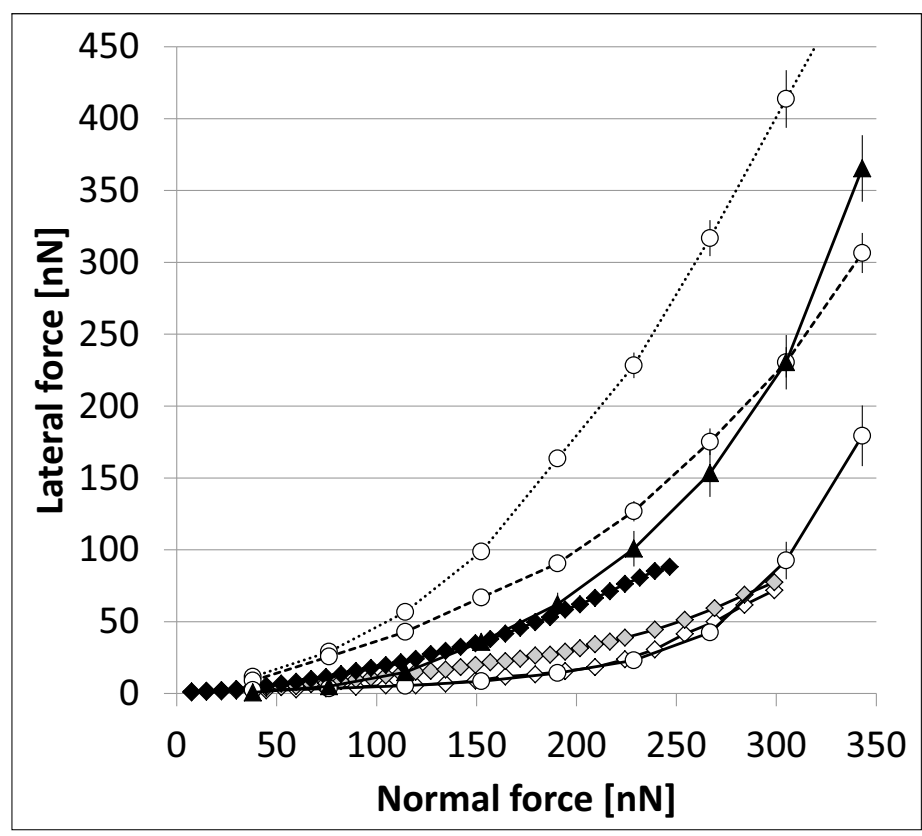

Fig. 3. Data from Fig. 1 and Fig. 2 overlaid for comparison. The friction response of the dense dextran brush in the presence of solutes (filled shapes) is comparable to the response of sparser dextran films in ultrapure water (broken lines).

response in ultrapure water at the lowest applied loads, but diverge at higher loads. A similar trend is already apparent in Fig. 2, which shows the local coefficient of friction diverging more rapidly from the initial value with increasing salt concentrations. Fig. 3 also shows that the dense brush in the presence of solutes exhibits a higher critical load than a sparser brush in water with similar friction values. This difference may reflect the contribution to the load-bearing capacity of the brush made by steric forces, which are only weakly affected by the presence of solutes.

\section{Conclusions}

We have demonstrated that the lubricity of dextran brushes can be influenced by solutes that reduce the strength of the interchain hydrogen bonding, without influencing the height or density of the brush in the absence of load. In particular, the solutes reduce the ability of the dextran brush to support high loads, while having less effect on the friction at low loads than a corresponding reduction in brush density, illustrating the relative contributions of interchain hydrogen bonding and steric forces to the lubricity of the brush as a function of applied load. This has implications for the model of physiological lubrication by saccharide-bearing, brush-like systems, which usually operate in the presence of much higher salt concentrations. In biological systems, the presence of other species that enhance the hydrogen bonding or directly cross-link the chains may serve to offset the failure of inter-chain hydrogen bonding under those conditions. Similar techniques might be used to extend the lubricious regime of the dextran brush to higher loads, or to tailor the inter-chain in- teractions for further applications such as trapping and then releasing biomolecules.

\section{Experimental}

\section{PLL-g-dextran Brushes}

The PLL-g-dex copolymer synthesis via reductive amination has been described previously.[8,9] Dextran (dextran T5, 5.2 $\mathrm{kDa}$, polydispersity 1.8, Pharmacosmos A/S, Denmark) was mixed with poly(Llysine)- $\mathrm{HBr}$ (20 kDa, polydispersity 1.1, Sigma-Aldrich, Switzerland) in a sodium borate buffer $(0.1 \mathrm{M}$, pH $8.5,0.4 \mathrm{M} \mathrm{NaCl})$. The terminal aldehydes of the dextran chains formed a Schiff base with the primary amines of the PLL, which was then reduced with sodium cyanoborohydride. The product was separated from the reactants by filtration with ultracentrifugation. The grafting ratio of lysine monomers to dextran chains was determined to be 5.3 via ${ }^{1} \mathrm{H}-\mathrm{NMR}$ in $\mathrm{D}_{2} \mathrm{O}$ and elemental analysis.

\section{Optical Measurements of Brush Thickness and Refractive Index}

Optical Waveguide Lightmode Spectrometry (OWLS, Microvacuum, Hungary) was used to monitor the adsorption of the brush and subsequent exposure to trehalose solution in real time. The standard $\mathrm{Si}_{0.75} \mathrm{Ti}_{0.25} \mathrm{O}_{2}$ chip was ultrasonicated in COBAS (Roche, Switzerland) for $10 \mathrm{~min}$, rinsed in ultrapure water, ultrasonicated in 2-propanol, dried in a nitrogen stream, and cleaned in a UV/ozone cleaner (Bioforce Nanosciences, Iowa, USA) for $30 \mathrm{~min}$. The cleaned waveguide was incubated in ultrapure water in the flow cell overnight to achieve a stable baseline. The solution of $0.02 \mathrm{~g} / \mathrm{L}$ PLL-g-dex in ultrapure water was then introduced, and the sample allowed to stabilize until the change was less than the instrument experimental drift, then rinsed with ultrapure water, allowed to stabilize again, and finally exposed to the $5.5 \mathrm{mM}$ trehalose solution and allowed to stabilize. The thickness and refractive index given by the instrument were used to calculate the optical mass with the de Feijter equation, ${ }^{[23]}$ assuming a refractive index increment $\mathrm{dn} / \mathrm{dc}$ of $0.131 \mathrm{~cm}^{3} / \mathrm{g}$. ${ }^{[8,9]}$ As the experiment is performed in aqueous conditions, the water associated with the polymer layer is not included in the measured optical mass, referred to as the 'dry mass'.

Transmission Interference Adsorption Spectroscopy (TInAS) ${ }^{[20,21]}$ was used to monitor the adsorption of the brush and subsequent exposure to $\mathrm{K}_{2} \mathrm{SO}_{4}$ solution in real time. A standard TInAS sensor, consisting of glass coated with $25 \mathrm{~nm} \mathrm{Al}$ and $3 \mu \mathrm{m} \mathrm{SiO}_{2}$, was ultrasonicated twice for $5 \mathrm{~min}$ in toluene, then twice for $5 \mathrm{~min}$ in 2-propanol, then dried with nitrogen and exposed to oxygen plasma (Plasma Cleaner/Sterilizer PDC-32G, Harrick, NY, USA) for $2 \mathrm{~min}$. The cleaned sensor was incubated in ultrapure water overnight to achieve a stable baseline. Then, the solution of $0.02 \mathrm{~g} / \mathrm{L}$ PLL-g-dex in ultrapure water was introduced, and the sample allowed to stabilize until the change was less than the instrument experimental drift, then rinsed with ultrapure water, allowed to stabilize again, and finally exposed to the $10 \mathrm{mM}$ $\mathrm{K}_{2} \mathrm{SO}_{4}$ solution and allowed to stabilize. The dry optical mass was calculated from the obtained thickness and refractive index in the same way as with the OWLS.

Variable Angle Spectroscopic Ellipsometry (VASE, J. A. Woolam Co, Inc., NE, USA) was used to check the adsorption of the brush onto the silicon wafers used for the friction measurements. Bare wafers were cleaned by the same procedure as the TInAS sensors, then rinsed in ultrapure water and dried with nitrogen. The thickness of the native oxide was measured in multiple places at wavelengths $370-1000 \mathrm{~nm}$ at an incident angle of $70^{\circ}$. The thickness variation over the $1 \mathrm{~cm}^{2}$ wafers was less than $0.5 \AA$. The substrates were then exposed for different lengths of time to the PLL- $g$-dex solutions, rinsed in ultrapure water, and dried with nitrogen. The dextran brush thickness was then measured in several places under the same conditions, and also showed variation over the wafers of less than $0.5 \AA$. In contrast to OWLS and TInAS, the VASE measurement is performed in air, rather than in aqueous solution, and therefore also includes any water associated with the film. Additionally, the effective refractive index of the film is not measured by the VASE and must be assumed. For these reasons, the measured thickness does not 
agree quantitatively with TInAS/OWLS measurements, and the adsorbed dry mass cannot be directly calculated. However, in previous work, ${ }^{[10]}$ the VASE thickness (assuming an effective refractive index of 1.45) was found to scale linearly with the TInAS/OWLS dry mass. Therefore the VASE thickness was multiplied by the scaling proportion to determine the dry optical mass on the wafers, and thereby the $\mathrm{L} / 2 \mathrm{R}$ values, of the dextran layers, with resulting values of $\mathrm{L} / 2 \mathrm{R}_{\mathrm{g}}$ ranging from 1.04 to 0.5 .

\section{Colloidal Probe Lateral Force Measurements}

The friction measurements were conducted using an MFP-3D AFM (Asylum Research, California, USA). Borosilicate colloidal spheres (Kromasil, Eka Chemicals $\mathrm{AB}$, Sweden; radius $\approx 8 \mu \mathrm{m}$ ) were glued to tipless rectangular cantilevers (CSC12, Mikromasch, Estonia) with UV-curable epoxy (Norland Optical Adhesive 63, Norland, NJ, USA). The glue was cured for 15 min under UV and then allowed to set overnight at $50{ }^{\circ} \mathrm{C}$. Prior to attaching the sphere, the normal and lateral spring constants of the sphere were calibrated from the power spectral density of the thermal noise fluctuations ${ }^{[24]}$ and the method of Sader et al. ${ }^{[25]}$ using the measured cantilever dimensions.

Each bare colloid was first slid against a bare substrate to ensure that no transient effects, such as initial colloidal wear or initial laser drift, could influence the measurements. Friction measurements were made in contact mode by sliding the probe perpendicular to the cantilever axis over a distance of $1 \mu \mathrm{m}$ at a speed of $1 \mu \mathrm{m} / \mathrm{s}$.
The trace minus retrace (TMR) signal was recorded, which is directly proportional to the friction force. ${ }^{[26]}$ Fifteen curves were taken for each load, after which the position on the sample was shifted slightly to avoid any sample wear and the load increased for the next step. To obtain the friction force, the TMR signal was multiplied by the calibration constant determined using the test probe method of Carpick et al. [27] The measurements shown in Fig. 1 were made in a single session without disturbing the cantilever or laser, and likewise for Fig. 2, so the calibration does not affect their relative values, but only facilitates the comparison between the two datasets, shown in Fig. 3, and with other studies.

\section{Acknowledgements}

The authors are grateful for financial support by the Swiss National Science Foundation.

Received: January 16, 2012

[1] S. Lee, N. D. Spencer, in 'Superlubricity', Elsevier Science B.V., Amsterdam, 2007, Ch. 21

[2] S. Lee, M. Müller, M. Ratoi-Salagean, J. Vörös, S. Pasche, S. M. de Paul, H. A. Spikes, M. Textor, N. D. Spencer, Tribol. Lett. 2003, 15, 231.

[3] W. H. Briscoe, S. Titmuss, F. Tiberg, R. K. Thomas, D. J. McGillivray, J. Klein, Nature 2006, 444, 191.

[4] D. Dowson, 'History of Tribology', Professional Engineering Publishing, London, 1998.

[5] Q. Yang, C. Kaul, M. Ulbricht, Langmuir 2010. 26, 5746

[6] M. Roba, M. Naka, E. Gautier, N. D. Spencer, R. Crockett, Biomaterials 2009, 30, 2072

[7] S. Lee, N. D. Spencer, Science 2008, 319, 575.

[8] C. Perrino, S. Lee, S. W. Choi, A. Maruyama, N. D. Spencer, Langmuir 2008, 24, 8850.
[9] C. Perrino, S. Lee, N. D. Spencer, Tribol. Lett. 2009, 33, 83

[10] K. J. Rosenberg, T. Goren, R. Crockett, N. D. Spencer, ACS Appl. Mater. Interfaces 2011, 3, 3020.

[11] J. Klein, in 'Fundamentals of Tribology and Bridging the Gap Between the Macro- and Micro/Nanoscales', Kluwer Academic Pub. Dordrecht, 2001, p. 177-198.

[12] U. Raviv, S. Giasson, N. Kampf, J.-F. Gohy, R. Jérôme, J. Klein, Nature 2003, 425, 163.

[13] B. Liberelle, S. Giasson, Langmuir 2008, 24, 1550 .

[14] F. Goujon, P. Malfreyt, D. J. Tildesley, Macromolecules 2009, 42, 4310 .

[15] B. Yu. Zaslavsky, T. O. Bagirov, A. A Borovskaya, N. D. Gulaeva, L. H. Miheeva, A. U. Mahmudov, M. N. Rodnikova, Polymer 1989, 30, 2104.

[16] B. Yu. Zaslavsky, A. U. Mahmudov, T. O. Bagirov, A. A. Borovskaya, G. Z. Gasanova, N. D. Gulaeva, V. Yu. Levin, N. M. Mestechkina, L. M. Miheeva, M. N. Rodnikova, Colloid Polym. Sci. 1987, 265, 548 .

[17] Y. Zhang, J. H. Han, J. Food Sci. 2006, 71, 253.

[18] Y. Zhang, J. H. Han, J. Food Sci. 2010, 75, 8.

[19] M. Heyden, E. Bründermann, U. Heugen, G Niehues, D. M. Leitner, M. Havenith, J. Am. Chem. Soc. 2008, 130, 5773.

[20] M. Heuberger, T. E. Balmer, J. Phys. D: Appl. Phys. 2007, 40, 7245.

[21] T. Sannomiya, T. E. Balmer, M. Heuberger, J. Vörös, J. Phys. D: Appl. Phys. 2010, 43, 405302

[22] Malvern Zetasizer Nano application note MRK839-01, 2007.

[23] J. A. De Feijter, J. Benjamins, F. A. Veer, Biopolymers 1978, 17, 1759.

[24] S. M. Cook, T. E. Schäffer, K. M. Chynoweth, M. Wigton, R. W. Simmonds, K. M. Lang, Nanotechnology 2006, 17, 2135.

[25] J. E. Sader, J. W. M. Chon, P. Mulvaney, Rev Sci. Instrum. 1999, 70, 3967.

[26] J. Ruan, B. Bhushan, J. Mater. Res. 1993, 8 3019.

[27] R. J. Cannara, M. Eglin, R. W. Carpick, Rev. Sci. Instrum. 2006, 77, 053701 